\title{
Les refus de délivrance de visas fondés sur une inscription au Système Information Schengen
}

\section{Claire Saas}

\section{OpenEdition}

12 Journals

\section{Édition électronique}

URL : http://journals.openedition.org/conflits/917

DOI : $10.4000 /$ conflits. 917

ISSN : $1777-5345$

Éditeur :

CCLS - Centre d'études sur les conflits lilberté et sécurité, L'Harmattan

\section{Édition imprimée}

Date de publication : 15 mars 2003

Pagination : 63-83

ISBN : 2-7475-5162-8

ISSN : 1157-996X

\section{Référence électronique}

Claire Saas, "Les refus de délivrance de visas fondés sur une inscription au Système Information Schengen », Cultures \& Conflits [En ligne], 50 | été 2003, mis en ligne le 29 septembre 2003, consulté le 30 mars 2021. URL : http://journals.openedition.org/conflits/917 ; DOI : https://doi.org/10.4000/ conflits. 917

Ce document a été généré automatiquement le 30 mars 2021.

Creative Commons License 


\title{
Les refus de délivrance de visas fondés sur une inscription au Système Information Schengen
}

\author{
Claire Saas
}

Les décisions de refus de délivrance de visas peuvent être fondées sur l'inscription du demandeur au système information Schengen (SIS). Cela résulte d'une part de la lettre de la Convention de Schengen. En effet, l'article $5^{1}$ prévoit que les parties contractantes sont susceptibles de refuser l'entrée sur le territoire pour un séjour n'excédant pas trois mois à un étranger signalé aux fins de non-admission. Pour sa part, l'article $96^{2}$ définit les circonstances dans lesquelles des données relatives à des étrangers peuvent être signalées sur le SIS. Ce mécanisme correspond d'autre part à l'esprit de Schengen : en contrepartie de l'ouverture des frontières intérieures à l'espace Schengen, il y a un renforcement des contrôles aux frontières extérieures. Le contrôle exercé par les Etats contractants l'est notamment lors de la délivrance des visas par le biais de la consultation du SIS.

La Convention et le droit interne français ont certes mis en place un système d'accès aux données inscrites dans le SIS, mais l'effectivité de cet accès demeure encore limitée, malgré quelques évolutions jurisprudentielles récentes. Si le contentieux portant sur les refus de délivrance de visas est devenu extrêmement riche suite à l'obligation faite à l'administration de motiver certains refus de visas - dont ceux fondés sur une inscription au SIS -, la mise en place de la Commission de recours contre les décisions de refus de visas d'entrée vise précisément à limiter ce contentieux. Malgré tout, des perspectives ont été encore ouvertes, notamment du fait de la prise en considération des droits fondamentaux issus de la Convention européenne des droits de l'homme. L'existence d'un contrôle juridictionnel supra-national exercé par la Cour de Justice des Communautés Européennes sur la Convention de Schengen semblait encore très lointaine lors de l'entrée en vigueur du traité d'Amsterdam. Ce contrôle commence pourtant à se mettre en place, promettant ainsi de nouvelles discussions portant 
notamment sur la conformité des dispositions intergouvernementales au droit communautaire.

Un accès aux données inscrites sur le SIS en progression

L'accès aux données inscrites sur le fichier informatique commun aux Etats Schengen est une question importante dans la mesure où la transparence des pratiques n'est pas ce qui caractérise véritablement l'usage du SIS. Or, pour contester une décision de refus de visa fondée sur une inscription au SIS, voire la décision d'inscription elle-même, la raison et la teneur du signalement sont fondamentales.

Lorsque les Etats ont commencé à intégrer des données dans le SIS, ils sont remontés très loin. Par exemple, un arrêté d'expulsion datant de 1973 a été à l'origine d'une inscription au SIS. De même, des arrêtés de reconduite à la frontière de 1985 ont été la cause de certains signalements. L'article 113 de la Convention de Schengen précise que les données relatives aux étrangers peuvent être conservées pour une durée maximale de dix années sur le support N-SIS. Cela signifie qu'une décision d'éloignement prise avant l'entrée en vigueur de la Convention de Schengen pouvait justifier une inscription sur le SIS en 1995, et que le signalement sera opposable à l'intéressé jusqu'en 2005.

Après leur effacement de ce fichier, les données peuvent être conservées durant une année dans la fonction de support technique, le C-SIS. Durant cette période, elles ne peuvent être consultées que pour le contrôlea posterioride leur exactitude et de la licéité de leur intégration. Ensuite, elles devront être effacées. Si aucun contrôle effectif n'a eu lieu alors que les données inscrites avaient un impact sur l'entrée et le séjour des personnes signalées, et ce pendant dix ans, on peut légitimement soulever l'intérêt d'un contrôle a posteriori sur des données qui sont censées ne plus produire d'effets.

Une décision prise par l'Allemagne, la Belgique ou l'Italie peut donc avoir en France une durée de vie fort longue, en termes d'effets sur la délivrance de visa ou sur l'entrée sur le territoire Schengen. Certes, la Convention de Schengen a mis en place les grandes lignes d'un dispositif d'accès, de vérification, voire d'effacement de ces données. Ainsi, son article 109 prévoit que toute personne a le droit d'accéder aux données la concernant qui sont intégrées dans le SIS, dans le respect du droit de l'Etat Schengen auprès de laquelle il le fait valoir. De plus, toute personne peut saisir l'autorité nationale compétente d'une action en rectification ou en effacement d'un signalement au SIS la concernant - article 111 - ou en vue de lui demander les données la concernant et l'utilisation qui en est faite - article 114.

Le texte de la Convention est clair. On comprend la possibilité d'obtenir la communication des informations concernant la personne signalée, à part si cette communication peut nuire à l'exécution du signalement ou si elle est nécessaire à la protection des droits et libertés d'autrui ou encore si l'Etat signalant a refusé la communication des informations. Le droit national de la partie contractante signalante s'applique au signalement, sauf conditions plus exigeantes prévues par la présente Convention et, pour autant qu'elle ne prévoit pas de dispositions particulières, le droit de chaque partie contractante est applicable aux données intégrées dans la partie nationale du SIS. Si la Convention détermine les droits reconnus aux personnes inscrites au SIS, c'est le droit interne qui détermine les modalités d'exercice de ces droits.

En droit français, il existait avant l'entrée en vigueur de la Convention de Schengen un système de protection des données et un encadrement du droit d'accès, de vérification 
et de rectification. Ce droit d'accès était indirect pour les fichiers de police conformément à l'article 39 de la loi du 6 janvier 1978 relative à l'informatique, aux fichiers et aux libertés. L'article 6 du décret du 6 mai 1995 confirme le fait que le droit d'accès est indirect pour le SIS, puisqu'il s'agit d'un fichier intéressant la sûreté de l'Etat, la défense et la sécurité publique. On voit ici l'impact du mélange des genres avec des inscriptions au SIS fondées sur des condamnations pénales et celles fondées sur une simple infraction à la législation sur les étrangers. Il faudra donc d'abord saisir la C.N.I.L., qui désignera un de ses membres, qui procédera aux démarches. A l'issue des démarches, celui-ci informera l'étranger que les vérifications nécessaires ont été effectuées. L'intérêt de cet accès indirect au SIS est donc très limité. Comment être sûr que toutes les diligences ont été assurées? Comment savoir si on est ou non inscrit au SIS ? Comment connaitre le motif de cette inscription?

Il semble bien que le texte de la Convention, qui prévoit la communication des données comme principe, soit bien plus favorable que l'article 39 de la loi du 6 janvier 1978. Cette différence entre le droit interne et la norme internationale apparaissait difficilement contestable. On voit mal, en effet, comment l'intéressé pourrait introduire un recours contre une décision individuelle qui, en elle-même, ne fait pas grief. Si une personne se voit refuser la délivrance d'un visa ou d'un titre de séjour au motif d'une inscription au SIS, on pourrait imaginer que le juge administratif saisi d'un recours pour excès de pouvoir exerce un contrôle de compatibilité entre le droit interne et la Convention de Schengen.

Il pourrait également être envisageable d'opposer au droit français la directive européenne du 24 octobre 1995 relative à la protection des personnes physiques à l'égard du traitement des données à caractère personnel et à la libre circulation des données. Cependant l'article 3-2 de la directive prévoit que ne relèvent pas du champ d'application de la directive toutes les activités qui ne relèvent pas du champ communautaire, telles que celles visées aux titres V et VI du TUE «et, en tout état de cause, les traitements ayant pour objet la sécurité publique, la défense, la sûreté de l'Etat (y compris le bien-être économique de l'Etat lorsque ces traitements sont liés à une question de sûreté de l'Etat) et les activités de l'Etat relatives à des domaines du droit pénal ». Il est donc fort possible que le SIS échappe à l'emprise des dispositions de la directive. Reste la question de la conformité du droit interne français avec le dispositif prévu par la Convention.

En novembre 2002, le Conseil d'Etat a rendu deux décisions fort intéressantes sur la question de l'accès aux données contenues dans le SIS $^{3}$ et a ainsi certainement montré le souci de respecter la lettre de la Convention. Si la haute juridiction estime que le système national d'accès aux données est compatible avec la Convention de Schengen, c'est au prix d'une interprétation quelque peu nouvelle de la notion du « droit d'accès indirect » : "parmi les informations relatives[aux requérants]et susceptibles de figurer dans le système informatique national du système d'information Schengen, certaines pourraient devoir[leur]être communiquées tandis que d'autres, qui mettent en cause les fins du traitement, ne seraient pas susceptibles de l'être». Le Conseil d'Etat revient donc sur le principe d'indivisibilité des fichiers : auparavant, si le fichier dans son ensemble concernait la sûreté de l'Etat, la défense ou la sécurité publique, l'ensemble des demandes de communications étaient soumises au droit d'accès indirect; désormais, les données dont la communication ne mettrait pas en cause les fins assignées au fichier informatisé feront l'objet d'un droit d'accès direct. Sont notamment concernées les 
"décisions administratives ou juridictionnelles qui ont été ou auraient dû préalablement être communiquées à l'intéressé». Dans la mesure où la loi française ne précise pas quelles sont les données auxquelles il serait alors possible d'avoir accès, le Conseil d'Etat s'en remet au juge administratif pour opérer lui-même la distinction entre les données communicables et celles qui ne le sont pas. Il introduit donc un contrôle de l'utilisation de l'article 39 de la loi du 6 janvier 1978, qui ne peut plus justifier ipso facto un accès indirect aux données contenues dans le SIS. Le Conseil d'Etat se rapproche ainsi du principe de communication des données enregistrées dans le SIS prévu par la convention, permettant notamment de s'assurer de la validité de l'inscription et des décisions qui peuvent en découler, tel un refus de délivrance de visas.

Un contentieux déjà riche

Les refus de délivrance de visas sont des décisions administratives individuelles qui, par dérogation à la loi du 11 juillet 1979 relative à la motivation des actes administratifs, n'avaient pas, pendant longtemps, à être motivés. Depuis la modification de l'article 5-1 ${ }^{\circ}$ de l'ordonnance du 2 novembre 1945 opérée par la loi Chevènement du 11 mai 1998, certains refus de visas doivent être motivés, dont ceux concernant des étrangers inscrits au SIS ${ }^{4}$. Même si l'obligation de motivation devait se résumer en la simple indication de l'existence d'un signalement au SIS, elle a le mérite d'épargner à l'intéressé des démarches lentes et jusqu'à présent peu fructueuses auprès de la C.N.I.L.

Le Conseil d'Etat, par deux décisions en date du 9 juin 1999, a infléchi de manière capitale la situation des personnes auxquelles on refuse la délivrance d'un visa en raison d'une inscription au SIS.

Dans la première affaire, Madame Hamssaoui demandait l'annulation de la décision du consul général de France à Casablanca lui refusant la délivrance d'un visa de séjour. Cette décision avait été motivée par le signalement de Mme Hamssaoui au SIS, se bornant à énumérer les dispositions applicables en l'espèce. Le Conseil d'Etat relève que la décision ne satisfait cependant pas à l'exigence de motivation posée par l'article 5 de l'ordonnance du 2 novembre 1945. Le fait d'indiquer l'inscription au SIS et la possibilité d'exercer un droit d'accès aux signalements auprès de la C.N.I.L. n'est pas une motivation suffisante. Il manque l'indication de l'autorité nationale qui a procédé au signalement de l'intéressée, "afin de permettre à la personne en cause d'exercer, le cas échéant, les recours qui lui appartiennent à l'encontre de la décision de signalement ».

L'exigence de motivation posée par l'article 5 de l'ordonnance du 2 novembre 1945 ne se réduit donc pas à la simple indication de l'inscription au SIS mais doit comporter l'indication de l'Etat-partie signalant afin de permettre l'exercice d'un recours effectif contre le signalement lui-même. Le fait de connaître et de s'adresser directement à l'autorité compétente de l'Etat signalant peut être déterminant pour débloquer la situation de l'intéressé. Si on s'adresse à la C.N.I.L., on a vu que les réponses demeurent très laconiques et ne permettent pas réellement d'avancer. En revanche, lorsqu'on saisit l'autorité compétente allemande, homologue de la C.N.I.L., les réponses sont substantielles et rendent compte des opérations effectuées et de leurs résultats.

Dans la seconde affaire, Monsieur et Madame Forabosco demandaient l'annulation du refus de délivrance d'un visa long séjour par le consul de France à Bucarest, qui avait été motivé par l'existence d'un signalement au SIS. On peut d'abord s'étonner du fait qu'un refus de visa long séjour puisse être motivé par une inscription au SIS, alors que la Convention de Schengen n'a vocation à régir que les visas court séjour. Le Conseil 
d'Etat balaie cet argument, en refusant de considérer que le consul de France à Bucarest s'est senti lié par l'inscription au SIS pour refuser le visa.

C'est certainement une manière pour le Conseil d'Etat de se laisser une porte ouverte afin de pouvoir se prononcer véritablement sur l'application et l'interprétation de la Convention de Schengen en France, et de définir plus précisément les pouvoirs du juge administratif français. Celui-ci est tenu de se prononcer sur le bien-fondé du moyen tiré du caractère injustifié d'un signalement - qui a motivé le refus de délivrance de visa alors même qu'il a été prononcé par une autorité administrative étrangère. Cela signifie, dans un premier temps, que le juge administratif doit être informé des motifs pour lesquels l'inscription a été effectuée et, dans un second temps, qu'il doit se prononcer sur la légalité même de l'inscription au SIS, même si elle émane d'une autorité étrangère. En l'espèce, Madame Forabosco avait été signalée au SIS par les autorités allemandes en tant que simple demandeur d'asile débouté, sans que cette décision de rejet soit accompagnée d'une interdiction d'entrée ou de séjour sur le territoire allemand. Le Conseil d'Etat estime que ce premier motif d'inscription ne figure pas dans les différents motifs énumérés par l'article 96 de la Convention de Schengen et que, par conséquent, l'inscription opérée par l'Allemagne, pour ce cas-là, est injustifiée.

Cette première partie de la décision est extrêmement intéressante à plusieurs égards. On aurait d'abord pu imaginer qu'une décision prise par un Etat-partie à Schengen - un signalement au SIS notamment - ne pouvait être remise en cause par les juridictions d'un autre Etat-partie, dans sa motivation tout au moins, et qu'elle devait être suivie d'effet, sauf exceptions et consultations préalables de l'autre Etat, conformément à l'idée de solidarité entre Etats qui présidait à la conclusion des accords de Schengen. On voit que le pouvoir du juge administratif est plus étendu qu'on ne pouvait s'y attendre.

Ensuite, en ce qui concerne l'espèce elle-même, à savoir que le rejet d'une demande d'asile ne fait pas partie des motifs figurant à l'article 96 qui pourraient justifier une inscription au SIS, la position prise par le Conseil d'Etat est également très intéressante. En effet, l'article 96 de la Convention de Schengen dresse une liste des motifs pouvant justifier une inscription au SIS. La rédaction de cette liste laisse penser que son contenu est indicatif, en aucun cas exhaustif. Pourtant, il semble que l'administration française ait apprécié cette liste comme étant limitative. En effet, les motifs d'inscription au SIS aux termes de l'alinéa 3 de l'article 96 sont, d'après le ministère de l'Intérieur, une interdiction d'entrée ou de séjour. Le rejet d'une demande d'asile en France n'entraîne pas une interdiction de séjour ou d'entrée, et ne saurait donc justifier en lui-même l'inscription au SIS d'un demandeur d'asile débouté.

Or, en Allemagne, le rejet d'une demande d'asile peut entraîner un signalement aux fins de non-admission, mais à l'issue d'une procédure tout à fait discutable. En Allemagne, le demandeur d'asile obtient un droit au séjour provisoire qu'il conserve jusqu'à ce que la procédure soit terminée, en raison soit du rejet de la demande, soit de son retrait. A la fin de la procédure d'asile, la personne déboutée n'est plus soumise aux règles sur l'asile, mais aux règles communes aux étrangers pour l'entrée et le séjour. Elle doit, par conséquent, quitter le territoire allemand. Même si elle dispose d'un délai pour quitter le territoire, elle se trouve en pratique en situation irrégulière. Ce séjour, qui est, en théorie, illégal ne peut pourtant pas motiver une inscription au SIS. En principe, on donne à l'intéressé un certificat de passage de frontière, sur lequel est inscrit le délai imparti pour quitter le territoire. A la frontière, l'intéressé doit remettre ce certificat 
aux officiers du BundesGrenzSchutz (BGS), qui le transmet à l'administration du Land, qui sait ainsi que l'intéressé s'est conformé à la décision relative à la demande d'asile.

Mais il peut arriver que l'étranger sorte du pays sans remettre le certificat de franchissement de la frontière, qu'il remette ce certificat mais que celui-ci ne soit pas transmis à l'administration, ou encore qu'il quitte le territoire sans attendre l'issue de l'examen de sa demande d'asile.

Dans ce cas de figure, l'administration part généralement du principe que le demandeur d'asile se trouve en situation irrégulière en Allemagne. Elle procède alors à l'inscription de l'intéressé dans le fichier central des étrangers, afin que les policiers arrêtent l'étranger et le reconduisent d'office à la frontière. Une telle inscription est conforme à la législation allemande. Et c'est à cette étape qu'une erreur est commise. En effet, l'article 8 paragraphe 2 de la loi sur les étrangers prévoit qu'un étranger ne peut pas revenir en Allemagne, lorsqu'il a été expulsé ou reconduit à la frontière. Mais seules ces deux mesures peuvent entraîner une interdiction du territoire allemand. Un étranger expulsé ou reconduit à la frontière sera inscrit au registre central des étrangers sous le mot clé « arrestation / expulsion / reconduite ».

Or, la plupart des administrations qui s'occupent des étrangers ont transféré et transfèrent dans le SIS la totalité des personnes inscrites sous le mot clé «arrestation / expulsion / reconduite", sans différencier les deux cas de figure, de manière automatique. C'est ainsi que de nombreux demandeurs d'asile déboutés, partis du territoire allemand, ont néanmoins été signalés au SIS, du fait de cette confusion.

Sous le même mot clé, on inscrit donc les étrangers dont on suppose qu'ils sont entrés dans la clandestinité et ceux qui doivent encore être reconduits à la frontière. Dans le premier cas de figure, il s'agit de ne pas laisser les personnes inscrites à nouveau entrer en Allemagne, tandis que, dans le deuxième cas de figure, il s'agit d'arrêter l'intéressé afin de le reconduire à la frontière. Il y a donc confusion des hypothèses et amalgame des situations.

On perçoit, à travers cette décision, toute la difficulté de l'application de la Convention de Schengen et de la possibilité pour le juge administratif de se prononcer sur des décisions étrangères. En principe, aux termes de l'article 111 paragraphe 2 de la Convention de Schengen, «les Parties contractantes s'engagent mutuellement à exécuter les décisions définitives prises par les juridictions ou autorités visées au paragraphe 1 \%. On retrouve ici l'idée selon laquelle les Etats parties à Schengen doivent Suvrer de manière coordonnée afin de lutter contre les menaces, en se faisant une confiance mutuelle, voire aveugle, sur la détermination de la menace. Une fois de plus, l'accent est mis sur la coordination des moyens de lutte contre les menaces, et non pas sur l'harmonisation des critères permettant d'adopter une définition commune de la menace. Or, les différences d'appréciation de la sécurité interne et des menaces à l'ordre public sont telles que la confiance mutuelle entre Etats est, comme nous l'avons vu, difficilement envisageable. La France peut-elle faire confiance aux autorités allemandes qui signalent des demandeurs d'asile déboutés au SIS, alors qu'elle-même ne le fait pas, et inversement? On peut évidemment tenter de suppléer à ce manque d'harmonisation par l'intermédiaire d'un contrôle juridictionnel étendu, à condition que celui-ci soit effectivement mis en Suvre. C'est cette position qu'a adopté le Conseil d'Etat en France, en contrôlant les motifs de signalement au SIS. Dans ce sens, les décisions du 9 juin 1999 sont réellement fondamentales.

De ces deux décisions, il faut garder à l'esprit : 
La décision de refus de délivrer un visa pour cause d'inscription au SIS n'est pas suffisamment motivée si les autorités consulaires se contentent d'indiquer que c'est «l'inscription au SIS qui a motivé cette décision ». La loi du 11 mai 1998 n'est donc pas dénuée d'effets.

Le refus de délivrance de visa, en raison d'une inscription au SIS, doit au moins comporter le nom de l'autorité signalante et le motif, afin que l'intéressé soit en mesure de vérifier que ce motif s'inscrit dans l'un des cas prévus à l'article 96 et qu'il puisse exercer un recours effectif.

De plus, la motivation de l'inscription doit être examinée par les juridictions, donc produite par l'administration consulaire, si le requérant invoque son caractère injustifié.

En cas d'inscription au SIS motivée par une décision qui constitue un motif d'inscription de l'article 96 de la Convention de Schengen pour la législation de l'Etat signalant mais qui n'en est pas un en droit français, l'inscription au SIS doit être considérée comme étant injustifiée. Il a été réaffirmé par le Conseil d'Etat que le rejet d'une demande d'asile ne peut constituer un motif de signalement sur le SIS par les autorités allemandes, dans la mesure où « un tel motif n'est pas au nombre de ceux qui sont limitativement énumérés aux 2 et 3 de l'article 96 de la convention d'application de Schengen pour justifier un signalement aux fins de non-admission $»^{5}$.

Cette jurisprudence fondatrice en la matière a été totalement suivie d'effets par le Conseil d'Etat dans ses décisions ultérieures, en exigeant notamment que les motifs de l'inscription au SIS soient systématiquement communiqués par le ministère des affaires étrangères en cas de litige quant à un refus de délivrance de visa fondé sur une telle inscription. Tel a encore été le cas dans une décision Kamate : "bien qu'il ait été invité à communiquer au Conseil d'Etat tous éléments relatifs au motif de ladite mesure[une mesure de signalement au SIS par l'Allemagne ayant justifié un refus de délivrance de visa par le consul général de France à Los Angeles],le ministère des affaires étrangères s'est borné à indiquer que, celle-ci ayant été supprimée par les autorités allemandes, il n'était pas à même d'en connaitre le motif, lequel n'était plus mentionné au SIS [\&], ainsi il n'a pas produit les éléments demandés, sans invoquer des considérations propres à l'espèce qui l'auraient empêché de satisfaire à ce qui lui avait été prescrit $»^{6}$.

Le contentieux relatif aux refus de délivrance de visas fondés sur une inscription au SIS est particulièrement riche. L'orientation choisie par le Conseil d'Etat a certainement été quelque peu modifiée du fait de deux réformes en droit administratif, l'une portant spécifiquement sur la question du contentieux des visas tandis que l'autre concerne plus largement les recours contentieux. Il s'agit de la création de la Commission de recours contre les refus de visa et le référé-suspension.

L'impact de la création de la Commission de recours contre les décisions de refus de visas d'entrée

Il est certainement nécessaire de remonter à la genèse de cette Commission de recours contre les décisions de refus de visa d'entrée, instituée par le décret $n^{\circ} 2000-1093$ du 10 novembre $2000^{7}$. Suite à la modification de l'ordonnance du 2 novembre 1945 rendant obligatoire la motivation des refus de délivrance de visas pour certaines catégories de personnes, le Conseil d'Etat, juridiction compétente en la matière, a très vite commencé à ployer sous le poids des recours contentieux dans ce domaine. Ainsi, au 31 décembre 2000 , les affaires en matière de visas représentaient $12 \%$ du nombre total des requêtes en suspens, qui s'élève à 16 189. Le Conseil d'Etata souhaité remédier à cet effet 
mécanique de la loi Chevènement, en exigeant la saisine préalable à tout recours contentieux contre un refus de visa d'une commission qui jouerait le rôle de filtre. Ainsi, tout recours contentieux qui n'aurait pas été précédé d'une saisine de la Commission, voire d'une décision de celle-ci serait déclaré irrecevable. Ce préalable est relativement dissuasif : il suppose, en effet, la connaissance de l'existence de cette commission et de sa saisine obligatoire, un allongement de la durée de la procédure et une complexification de celle-ci.

A l'issue d'un entretien, il s'avère que la commission de recours estime que la décision des autorités consulaires est fondée dans neuf cas sur dix. Si les intéressés décident de poursuivre devant le Conseil d'Etat malgré cet avis défavorable, le rôle initial de filtre que devait jouer cette commission ne sera absolument pas effectif dans une perspective de désengorgement de la juridiction administrative. En revanche, on peut redouter que l'examen par le Conseil d'Etat des arguments présentés par les requérants soit plus lapidaire en cas d'avis défavorable préalable de la Commission. Pour finir, la Commission n'est tenue de motiver son avis que s'il concerne l'un des cas prévus par l'article 5 de l'ordonnance du 2 novembre $1945^{8}$.

Les perspectives du référé-suspension

La seconde réforme concerne la procédure du référé-suspension, réalisée le 1er janvier 2001. Aux termes de l'article L 521-1 du code de justice administrative modifié par la loi du 30 juin 2000 : «quand une décision administrative, même de rejet, fait l'objet d'une requête en annulation ou en réformation, le juge des référés, saisi d'une demande en ce sens, peut ordonner la suspension de l'exécution de cette décision, ou de certains de ses effets, lorsque l'urgence le justifie et qu'il est fait état d'un moyen propre à créer, en l'état de l'instruction, un doute sérieux quant à la légalité de la décision».

Dans la mesure où le Conseil d'Etat ne peut désormais être valablement saisi d'un refus de visa que si le recours devant la Commission de recours contre les refus de visa a été exercé de manière préalable, le référé-suspension portant sur un refus de visa est subordonné à la saisine préalable de la Commission. Cela dit, il n'est pas nécessaire que cette dernière ait statué. Si la suspension est prononcée, elle vaut jusqu'à ce que la Commission ait statué. Si sa décision est négative, il faudra formuler une nouvelle demande de référé-suspension.

Deux conditions sont posées pour que le juge administratif décide la suspension de l'exécution d'une décision : il faut que l'urgence le justifie et qu'il soit fait état d'un moyen propre à créer un doute sérieux quant à la légalité de la décision.

Il faut noter la possibilité pour le juge administratif, s'il décide d'ordonner la suspension, de faire peser un certain nombre d'obligations sur le service administratif concerné, en vertu de son pouvoir général d'injonction. Ainsi, il pourra "assortir le prononcé du sursis de l'indication des obligations qui en découleront pour l'administration et qui pourront consister à réexaminer la demande dans un délai déterminé ou, le cas échéant, à prendre toute mesure conservatoire utile prescrite par le juge compte tenu de l'objet du litige, du moyen retenu et de l'urgence $»^{10}$.

Dans la mesure où cette faculté est maintenant également ouverte au juge administratif également en cas de décisions de rejet, les décisions de refus de visa peuvent être concernées par le référé-suspension, ainsi que par l'une des injonctions des articles L 911-1, L 911-2 et L 911-3 du code de justice administrative ${ }^{11}$. Il suffirait, pour ce faire, d'établir une situation d'urgence et d'apporter un moyen propre à créer un doute sérieux quant au refus de légalité du visa. En ce qui concerne la condition de l'urgence, 
on pourrait admettre une telle situation pour « une personne qui risquerait dans son pays des traitements inhumains et dégradants au sens de l'article 3 de la Convention européenne des droits de l'homme" ; de même si la présence de l'intéressé sur le territoire français est indispensable pour des raisons liées à son activité - commerçant, artiste - , pour l'examen de sa situation devant les juridictions françaises - réexamen du degré d'invalidité d'une personne en vue de la réévaluation de sa pension - ou encore pour le respect de sa vie privée et familiale au sens de l'article 8 de la Convention européenne des droits de l'homme - mariage, décès, naissance d'un membre de famille en France. Quant à la nécessité d'apporter un moyen de nature à créer un doute sérieux sur la légalité de la décision, tous les moyens de droit traditionnels sont envisageables: erreur de droit, erreur manifeste d'appréciation, violation de dispositions internationales.

Cette réforme de la procédure des référés en droit administratif a été accompagnée de la création du référé-liberté, conformément à l'article L 521-2 du code de justice administrative. Le recours à cette procédure est également envisageable en cas de refus de visa, à condition que l'exécution de cette décision entraine des conséquences difficilement réparables et qu'elle porte atteinte à une liberté fondamentale. A été jugé par le Conseil d'Etat, en tant que juge des référés, dans une décision Deperthes du 9 janvier 2001, que la liberté d'aller et venir constituait une liberté fondamentale, au même titre que le droit de demander l'asile.

Les procédures du référé-suspension et du référé-liberté visent toutes deux à réparer les atteintes à un droit fondamental. Et ce sont effectivement les droits fondamentaux reconnus par la Convention européenne des droits de l'homme qui ouvrent de nouvelles perspectives au contentieux liés au refus de délivrance de visas en France.

Les perspectives ouvertes par la Convention européenne des droits de l'homme

Parmi les moyens susceptibles d'entraîner la suspension ou l'annulation de la décision de refus de visa figurent les stipulations de la Convention européenne de sauvegarde des droits de l'homme, et notamment ses articles 3 (prohibition de la torture et des traitements inhumains et dégradants) et 8 (protection de la vie privée et familiale).

La question posée est la suivante: une stipulation de la Convention européenne des droits de l'homme peut-elle faire échec au principe de refus de délivrance d'un visa fondé sur une inscription au SIS ? Elle a été effleurée par le Conseil d'Etat dans une affaire X du 6 octobre 1999. En effet, le requérant avait fait valoir la contradiction de la décision avec l'article 8 de la Convention européenne des droits de l'homme. Finalement, la décision avait été annulée au motif que le ministère des affaires étrangères n'ayant pas produit tous les éléments relatifs à l'inscription de M. X au SIS, "le moyen du requérant tiré de ce que la mesure litigieuse est fondée sur un motif matériellement inexact [devait] être regardé comme établi ».

Certaines décisions ultérieures du Conseil d'Etat ont reconnu que Convention de Schengen et Convention européenne des droits de l'homme devaient être conciliées, ainsi qu'il résulte de l'article 5 paragraphe 2 de la Convention de Schengen, aux termes duquel « l'entrée sur le territoire des parties contractantes doit être refusée à l'étranger qui ne remplit pas l'ensemble de ces conditions, sauf si une partie estime nécessaire de déroger à ce principe pour des motifs humanitaires ou d'intérêt national ou en raison d'obligations internationales ».

Ainsi, dans plusieurs espèces concernant des refus de visa suite à une inscription au SIS $^{12}$, le Conseil d'Etat a sursis à statuer, s'estimant insuffisamment informé des motifs 
du signalement pour pouvoir examiner la proportionnalité entre l'atteinte portée au droit des requérants au respect de leur vie privée et familiale par le biais de la décision de refus et les motifs justifiant ce refus. Les décisions sur le fond n'ont pas été rendues, mais elles laissent entendre que les stipulations de la Convention européenne de sauvegarde des droits de l'homme peuvent être opposées à celles de la Convention de Schengen et donc, le cas échéant, peuvent y faire échec.

Plusieurs décisions du Conseil d'Etat ont d'ailleurs été rendues dans ce sens. Ainsi, dans une affaire $\mathrm{El} \mathrm{Abd}{ }^{13}$, le Conseil d'Etat a fait appel de manière explicite à l'article 8 de la Convention européenne des droits de l'homme. Une ressortissante marocaine s'était vu refuser la délivrance d'un visa court séjour, qu'elle sollicitait pour rendre visite à ses fils résidant en France, au motif que ses ressources étaient insuffisantes. Après avoir rappelé que la délivrance des visas ne rentre pas dans le cadre de compétences liées des autorités consulaires, le Conseil d'Etat décide d'annuler la décision de refus de visa au motif que « la décision présentement attaquée a porté au droit de la requérante au respect de sa vie privée et familiale une atteinte excessive par rapport aux buts en vue desquels cette décision a été prise ; que le consul général de France à Rabat a ainsi méconnu les stipulations de l'article 8 de la Convention européenne de sauvegarde des droits de l'homme et des libertés fondamentales ». Cela ne signifie pas, bien au contraire, que l'article 8 prévaudra, dans tous les cas de figure. Mais, dans chaque espèce, un argument tiré d'un principe posé par la Convention européenne des droits de l'homme sera examiné et mis en balance avec les stipulations de la Convention de Schengen ${ }^{14}$. Il a été notamment décidé que le refus de délivrer un visa à un ressortissant algérien, signalé sur le SIS en raison d'une mesure d'expulsion datant de 1973, afin qu'il puisse rendre visite à sa mère, invalide et âgée, résidant en France, portait atteinte au droit au respect de sa vie familiale ${ }^{15}$.

La recevabilité des arguments tirés de traités internationaux, et tout particulièrement de la Convention européenne des droits de l'homme, en relation avec les procédures du référé-suspension et du référé-liberté ouvrent de nouvelles perspectives au contentieux relatif aux refus de visa en France. Un ensemble de décisions commence donc à structurer les réponses aux questions posées par l'application de la Convention de Schengen. Il s'agit pour l'instant d'un ensemble national, propre à chaque pays, qui pourra certainement être influencé par l'existence d'un contrôle juridictionnel supranational.

Vers un contrôle juridictionnel supra-national ?

Bien que le contrôle juridictionnel soit, en France, assez performant, le risque d'interprétations et de décisions contradictoires demeure. Il suffit, pour s'en convaincre, de lire une décision d'une juridiction autrichienne relative à une décision de non-admission fondée sur un signalement au SIS suite à une décision d'expulsion prises par les autorités italiennes. Le requérant contestait la validité de la décision de refus d'entrée, au motif qu'elle était fondée sur une inscription au SIS justifiée par une mesure d'expulsion dont il contestait la validité, n'ayant jamais séjourné en Italie. La juridiction autrichienne rejette la requête de l'intéressé et précise notamment qu'aucune autorité, qu'il s'agisse de la police des frontières ou d'une juridiction administrative, n'a à vérifier que la décision d'expulsion prises par les autorités italiennes est légale. A partir du moment où l'intéressé est signalé au SIS, on doit partir du principe qu'il existe effectivement un motif de non-admission. Entre ce contrôle $a$ minima de la juridiction autrichienne et le contrôle étendu de la juridiction française, toute une palette de jurisprudence est envisageable. L'exercice d'un contrôle 
juridictionnel par une instance internationale serait ainsi d'un intérêt réel, afin d'éviter une telle mosaïque de jurisprudence.

Avec l'intégration de l'acquis Schengen en droit communautaire prévue par le Traité d'Amsterdam, la compétence de la Cour de Justice des Communautés Européennes devenait envisageable par l'intermédiaire de questions préjudicielles très certainement relatives au SIS. Cette compétence n'allait pas de soi. En effet, l'intégration de l'acquis Schengen s'est faite de manière diverse selon les matières. Les Etats membres ne s'étant pas mis d'accord sur l'intégration du SIS au premier pilier, celui-ci ressort du troisième pilier. Or, en vertu de l'article $35 \mathrm{du} \mathrm{TUE}^{16}$, la Cour de Justice des Communautés Européennes est compétente pour juger de la validité et interpréter les conventions négociées dans le cadre du troisième pilier, à condition que les Etats membres aient reconnu expressément cette compétence par le biais d'une déclaration.

Par des déclarations concomitantes à l'entrée en vigueur du Traité d'Amsterdam ${ }^{17}$, une majorité d'Etats membres ont reconnu la compétence de la Cour de Justice des Communautés Européennes pour statuer sur les questions relatives au troisième pilier, et notamment au SIS. La Cour doit être saisie au titre de questions préjudicielles, conformément aux termes de l'article $68 \mathrm{TCE}^{18}$. L'Espagne a précisé que seules les juridictions statuant en dernier ressort avaient la possibilité de poser une question préjudicielle, tandis que l'Allemagne, l'Autriche, la Belgique, la Finlande, la Grèce, l'Italie, le Luxembourg, les Pays-Bas, le Portugal et la Suède ont reconnu cette faculté à toutes les juridictions quel que soit leur ressort. Certains Etats, enfin, ont précisé que les juridictions statuant en dernier ressort seraient tenues de saisir la Cour d'une question préjudicielle: il s'agit de l'Allemagne, l'Autriche, la Belgique, l'Espagne, l'Italie, le Luxembourg et les Pays-Bas.

Tous les Etats membres faisant partie du dispositif Schengen avaient par conséquent reconnu la compétence de la Cour de Justice, au jour de l'entrée en vigueur du Traité d'Amsterdam, à l'exception notable de la France, qui demeure isolée dans son refus. On notera que cet isolement est d'autant plus incompréhensible que les juridictions françaises vont être, de toutes les manières et assez prochainement, amenées à respecter la jurisprudence de la Cour de Justice sur les questions relatives à Schengen.

Par ailleurs, la Commission a, dans une communication du 18 décembre 2001 portant sur le développement du Système Information Schengen II, estimé que le SIS a une nature mixte. Il s'agit effectivement d'un instrument de la coopération policière et judiciaire, qui relève donc du troisième pilier. Mais il est également utilisé dans des domaines relevant du premier pilier, comme en matière de visas. Par conséquent, le SIS aurait un double fondement juridique et s'inscrirait à la fois dans le premier et le troisième pilier. La compétence de la Cour de Justice des Communautés Européennes pour connaître des questions touchant au SIS ne serait donc plus problématique, y compris pour la France.

La première question préjudicielle touchant au dispositif Schengen a été portée devant la Cour de Justice par une juridiction allemande fin avril 2001 ${ }^{19}$. Elle porte sur l'interprétation de l'article 54 de la Convention de Schengen, relatif au principe non bis in idem, et concerne les conditions dans lesquelles l'extinction du droit de poursuite pénale "Transactie ", connu en droit néerlandais et mettant définitivement fin aux poursuites pénales sans l'intervention du juge, doit être considéré comme ayant donné lieu à une décision pénale définitive, empêchant qu'une personne soit jugée à raison des mêmes faits. Une seconde question préjudicielle relative à l'interprétation de 
l'article 54 ayant été posée par une juridiction belge début octobre $2001^{20}$, la Cour a décidé de joindre les deux affaires en raison de leur connexité et a rendu sa première décision portant sur la Convention de Schengen en février 2003 ; la question de la compétence de la Cour n'a par ailleurs été soulevée que par l'avocat général.

Il est donc probable qu'elle aura très prochainement à se prononcer sur les questions relatives au SIS et à la délivrance de visas. Tel sera peut-être le cas à la suite de la décision de la Commission européenne de poursuivre une procédure d'infraction contre l'Espagne. Sont concernées les décisions de refus de visa et de refus d'entrée sur le territoire prises à l'encontre de membres de famille de citoyens de l'Union européenne au motif qu'ils faisaient l'objet d'un signalement aux fins de non-admission au SIS. Si la Commission décide de saisir la Cour de Justice des Communautés Européennes, la validité de décisions de refus de visa et d'entrée fondées sur un signalement au SIS sera posée en regard du principe de libre circulation des communautaires et des membres de leur famille qui ne peut être limitée que dans des conditions conformes à la directive 64/221, lorsque l'intéressé constitue une menace réelle et suffisamment grave pour l'ordre public.

Un contrôle juridictionnel supra-national exercé par la Cour de Justice des Communautés Européennes sur la Convention de Schengen se met donc en place, ouvrant ainsi de nouvelles discussions sur l'application de la Convention de Schengen par les Etats membres. Espérons que le contrôle exercé par la Cour de Justice soit à la hauteur des exigences posées par la jurisprudence du Conseil d'Etat.

\section{NOTES}

1. . L'article 5(1) de la Convention de Schengen de 1990 stipule que : « Pour un séjour n'excédant pas trois mois, l'entrée sur le territoire des Parties contractantes peut être accordée à l'étranger qui remplit les conditions ci-après :

(a) Posséder un document ou des documents valables permettant le franchissement des frontières, déterminés par le Comité exécutif ;

(b) Etre en possession d'un visa valable si celui-ci est requis ;

(c) Présenter, le cas échéant, les documents justifiant de l'objet et des conditions du séjour envisagé et disposer de moyens de subsistance suffisants, tant pour la durée du séjour envisagé que pour le retour dans le pays de provenance ou le transit vers un Etat tiers dans lequel son admission est garantie, ou être en mesure d'acquérir légalement ces moyens ;

(d) Ne pas être signalé aux fins de non-admission ;

(e) Ne pas être considéré comme pouvant compromettre l'ordre public, la sécurité nationale ou les relations internationales de l'une des Parties contractantes ».

2. . L'article 96 de la Convention de Schengen stipule que : « (1) Les données relatives aux étrangers qui sont signalés aux fins de non-admission sont intégrées sur la base d'un signalement national résultant de décisions prises, dans le respect des règles de procédure prévues par la législation nationale, par les autorités administratives ou les juridictions compétentes. 
(2) Les décisions peuvent être fondées sur la menace pour l'ordre public ou la sécurité et sûreté nationales que peut constituer la présence d'un étranger sur le territoire national.

Tel peut être notamment le cas :

(a) D'un étranger qui a été condamné pour une infraction passible d'une peine privative de liberté d'au moins un an ;

(b) D'un étranger à l'égard duquel il existe des raisons sérieuses de croire qu'il a commis des faits punissables graves, y inclus ceux visés à l'article 71, ou à l'égard duquel il existe des indices réels qu'il envisage de commettre de tels faits sur le territoire d'une Partie contractante.

(3) Les décisions peuvent être également fondées sur le fait que l'étranger a fait l'objet d'une mesure d'éloignement, de renvoi ou d'expulsion non rapportée ni suspendue comportant ou assortie d'une interdiction d'entrée, ou, le cas échéant, de séjour, fondée sur le non-respect des réglementations nationales relatives à l'entrée ou au séjour des étrangers ».

3. . CE, M. Moon, 6 novembre 2002.

4. . L'article 5 de l'ordonnance du 2 novembre 1945 dispose que : «Par dérogation aux dispositions de la loi $\mathrm{n}^{\circ}$ 79-587 du 11 juillet 1979 relative à la motivation des actes administratifs et à l'amélioration des relations entre l'administration et le public, les décisions de refus de visa d'entrée en France, prises par les autorités diplomatiques ou consulaires, ne sont pas motivées sauf dans les cas où le visa est refusé à un étranger appartenant à l'une des catégories suivantes et sous réserves de considérations tenant à la sûreté de l'Etat : [...] - personnes faisant l'objet d'un signalement aux fins de nonadmission au Système d'Information Schengen ». Le signalement du demandeur au SIS n'entraîne pas automatiquement la motivation du refus de visa si la décision de l'autorité est fondée sur un autre motif.

5. . CE, M. Natchev, 9 janvier 2002.

6. CE, Kamate, 29 juillet 2002 ; voir également CE, Guetarni, 29 juillet 2002.

7. . Voir également le décret du 10 janvier 2001 relatif à la composition de la Commission.

8. . CE, Melle Zahia B., 27 novembre 2002.

9. . CE, Sté Produits Roche, 12 octobre 2001.

10. . CE, M. Ouatah, 20 décembre 2000.

11. . Cela dit, le Conseil d'Etat a rendu le 14 mars 2001 un arrêt de section «Mme Ameur » dans lequel il énonce que la condition d'urgence pour obtenir la suspension d'un refus de titre de séjour ne sera remplie que dans deux hypothèses :

1) en cas de refus de renouvellement de titre ou de retrait

2) dans les autres cas, il faudra justifier de « circonstances particulières caractérisant la nécessité (...) de bénéficier à très bref délai d'une mesure provisoire ».

12. . CE, Mme Cucicea-Lamblot, 25 octobre 2000 ; CE, M. Bolatdemirci, 29 décembre 2000.

13. . CE, Mme El Abd, 20 décembre 2000.

14. . Tel a été notamment le cas dans les affaires suivantes: CE, M. Rusu, 27 novembre 2000 ; CE, M. Sengül, 29 novembre 2000 ; CE, Mme Heddi, 17 novembre 2000 ; CE, 19 février 2001, Azouzuateh ; CE, Lekhlef, 23 novembre 2001 ; CE, Abla, 3 juin 2002 ; CE, Sellami, 14 juin 2002 ; CE, Azoum, 14 juin 2002 ; CE, Ouaaziz, 22 juin 2002.

15. . CE, Lekhlef, 23 novembre 2001.

16. . Article 35 (ex-article K.7) : 
1./ La Cour de justice des Communautés européennes est compétente, sous réserve des conditions définies au présent article, pour statuer à titre préjudiciel sur la validité et l'interprétation des décisions-cadres et des décisions, sur l'interprétation des conventions établies en vertu du présent titre, ainsi que sur la validité et l'interprétation de leurs mesures d'application.

2./ Tout Etat membre peut, par une déclaration faite au moment de la signature du traité d'Amsterdam, ou à tout autre moment postérieur à ladite signature, accepter la compétence de la Cour de justice pour statuer à titre préjudiciel dans les conditions définies au paragraphe 1.

3./ Un Etat membre qui fait une déclaration au titre du paragraphe 2 indique que : a) soit toute juridiction de cet Etat dont les décisions ne sont pas susceptibles d'un recours juridictionnel de droit interne a la faculté de demander à la Cour de justice de statuer à titre préjudiciel sur une question soulevée dans une affaire pendante devant elle et portant sur la validité ou l'interprétation d'un acte visé au paragraphe 1, lorsqu'elle estime qu'une décision sur ce point est nécessaire pour rendre son jugement,

b) soit toute juridiction de cet Etat a la faculté de demander à la Cour de justice de statuer à titre préjudiciel sur une question soulevée dans une affaire pendante devant elle et portant sur la validité ou l'interprétation d'un acte visé au paragraphe 1 , lorsqu'elle estime qu'une décision sur ce point est nécessaire pour rendre son jugement.

Traité sur l'Union européenne 25

4./ Tout Etat membre, qu'il ait ou non fait une déclaration au titre du paragraphe 2, a le droit de présenter à la Cour des mémoires ou observations écrites dans les affaires dont elle est saisie en vertu du paragraphe 1.

5./ La Cour de justice n'est pas compétente pour vérifier la validité ou la proportionnalité d'opérations menées par la police ou d'autres services répressifs dans un Etat membre, ni pour statuer sur l'exercice des responsabilités qui incombent aux Etats membres pour le maintien de l'ordre public et la sauvegarde de la sécurité intérieure.

6./ La Cour de justice est compétente pour contrôler la légalité des décisions-cadres et des décisions lorsqu'un recours est formé par un Etat membre ou par la Commission pour incompétence, violation des formes substantielles, violation du présent traité ou de toute règle de droit relative à son application, ou détournement de pouvoir. Les recours prévus au présent paragraphe doivent être formés dans un délai de deux mois à compter de la publication de l'acte.

7./ La Cour de justice est compétente pour statuer sur tout différend entre Etats membres concernant l'interprétation ou l'application des actes adoptés au titre de l'article 34, paragraphe 2, dès lors que ce différend n'a pu être réglé au sein du Conseil dans les six mois qui ont suivi la saisine de celui cci par l'un de ses membres. La Cour est également compétente pour statuer sur tout différend entre Etats membres et la Commission concernant l'interprétation ou l'application des conventions établies en vertu de l'article 34, paragraphe 2, point d).

17. . JOCE L 114, 1.5.1999, page 56

18. . 1./ L'article 234 est applicable au présent titre dans les circonstances et conditions suivantes : lorsqu'une question sur l'interprétation du présent titre ou sur la validité et l'interprétation des actes pris par les institutions de la Communauté sur la base du présent titre est soulevée dans une affaire pendante devant une juridiction nationale 
dont les décisions ne sont pas susceptibles d'un recours juridictionnel de droit interne, cette juridiction, si elle estime qu'une décision sur ce point est nécessaire pour rendre son jugement, demande à la Cour de justice de statuer sur cette question.

2./ En tout état de cause, la Cour de justice n'est pas compétente pour statuer sur les mesures ou décisions prises en application de l'article 62, point 1), portant sur le maintien de l'ordre public et la sauvegarde de la sécurité intérieure.

3./ Le Conseil, la Commission ou un Etat membre a la faculté de demander à la Cour de justice de statuer sur une question d'interprétation du présent titre ou d'actes pris par les institutions de la Communauté sur la base de celui-ci. L'arrêt rendu par la Cour de justice en réponse à une telle demande n'est pas applicable aux décisions des juridictions des Etats membres qui ont force de chose jugée.

19. . CJCE, Procédure pénale contre Hüseyn Gözütok, affaire C-187/01, 11 février 2003, conclusions de l'avocat général le 19 septembre 2002.

20. . CJCE, Procédure pénale contre Klaus Brügge, affaire C-385/01, 11 février 2003, conclusions de l'avocat général le 19 septembre 2002.

INDEX

Mots-clés : bases de données, Visa

Thèmes : Schengen (convention) 